\title{
CHAOS COMPUTING: AN INTRODUCTION
}

\author{
Matthew N. O. Sadiku, Adebowale E. Shadare, and Sarhan M. Musa \\ Roy G. Perry College of Engineering \\ Prairie View A\&M University \\ Prairie View, TX 77446
}

U.S.A

\begin{abstract}
There has been interest in so called alternative or unconventional computing paradigms that differ from traditional silicon-based computing architectures. One such paradigm is chaos computing. Chaos computing is based on the nonlinear dynamical principles and can perform all logical operations. It aims at implementing computing functions through the construction of logical gates by employing chaotic elements. The paper provides a brief introduction to chaos computing.
\end{abstract}

Key words: Chaos computing, Chaos-based computer.

\section{INTRODUCTION}

Computing for the last fifty years is based on Moore's law, which is now delivering diminishing returns. Modern digital computers perform computations based upon digital logic gates, which are essentially seven: AND, OR, NOT, NAND, NOR, XOR and XNOR. Chaos computing is a new unconventional paradigm for computing, where chaotic oscillators are used for computation. The word "chaos" commonly means a state of disorder, the existence of random behavior, utter confusion or disorganization. In mathematics, chaos signifies that the equations expressing nonlinear systems are extremely sensitive to initial inputs or conditions and it is hardly possible to predict their future behavior with a high degree of certainty [1].

Chaos has three defining characteristics [2]: (1) sensitivity to initial conditions, (2) aperiodic long-term behavior, and (3) it is wholly deterministic. Chaotic behavior is manifested in many natural systems, such as lasers, neurons in the brain, electronic circuits, chemical systems, weather, and road traffic

\section{CONCEPT OF CHAOS COMPUTING}

Choas computing uses chaotic systems for computation. Chaotic systems can be made to produce all types of logic gates. By controlling how inputs are mapped to outputs, a specific task can be performed. Chaotic computer can directly perform basic numerical operations like addition and multiplication. Research has shown how chaotic computers can be recruited in fault tolerant applications by introducing dynamic based fault detection methods [3]. A chaotic system enables us to build better computers that have a flexible instruction set and carry out computation that conventional computers are not good at.

The term "chaos computing" was introduced in 1975 by the US mathematicians Tien lien Li and James A. Yorke in their ground breaking paper, "Period Three Implies Chaos." They introduced a new direction to computing using nonlinear chaotic dynamics. The term "chaos computing" was popularized by William Ditto of the Georgia Institute of Technology, who believes that the unpredictabilty of chaotic processes may power a new breed of computer. Ditto's chip is like the microelectronic version of a stem cell that can assume all sorts of different functions. 
Choas computing takes advantage of the richness of nonlinear dynamical systems and insights from neural systems to devise new approaches to create a chaos-based computer. Nonlinear dynamics has revealed a rich array of behaviors, especially those related to chaos including routes to chaos, high and low dimensional chaotic attractors, and transient chaos. In neural systems, measured phenomena include chaos, synchrony, and cascading avalanches demonstrating that information processing in the brain is not just anatomical, but also dynamical.

A system is capable of serving as a universal general purpose computing provided it can emulate all logic gates. Using single chaotic element, each logical operation (AND, OR, NOT, NAND, NOR, XOR, ) can be realized [4]. A typical circuit for realizing NOR gate is shown in Figure 1 [5]. Computer memory can be constructed by combining logical gates.

Chaos computing can be applied for generic computation, DNA computing, modeling speech, and speech processing. It can also be implemented in specialized domains such as a biological environment and nano fluidics.

\section{BENEFITS AND CHALLENGES}

A reconfigurable chaos-based computer will be of great benefit to military and civilian customers because reuse of logic morphable gates can led to fewer logic gates and lower cooling requirements. The major benefit of a chaotic system is that it enables us to: (1) build better computers that have a flexible instruction set, and (2) carry out computation that conventional computers are not good at [6]. Other major potential advantages of chaos computing include high speed, low power, low cost, a general-purpose form of computing, dynamic logical architecture, implementation of continuous logic, robustness against noise, and parallel and distributed computing [7]. Using chaos based logic provides opportunities for building computing systems with similar hardware but different configurations of operation. A completely different circuit could be implemented on the same hardware. Chaos based architectures can help us avoid trillion dollar research and development that has only marginal gains.

Novel chaos computing can lead to new paradigms for the optimization of solving complex problems, such as controllers for autonomous systems, including robots and vehicles. A number of challenges may hinder the development of practical chaos-based computers. One major challenge for developing chaos-based computers is that the overhead associated with the physical implementation of a chaotic gate is quite higher than an equivalent digital gate [8].

\section{CONCLUSION}

Chaos computing is an unconventional computing paradigm that exploits the non-linearity of chaotic systems. The dynamic chaotic computer can perform computations and is promising because of its flexibility for changing logic gates by slightly modifying its parameter values. Chaos computing is still in its infancy. Chaos computing/theory remains an active area of research that involves several disciplines including mathematics, natural sciences ( physics, biology, and chemistry), psychology, meteorology, and robotics More information on chaos computing can be found in the book in [9].

\section{REFERENCES}

[1] "Chaos theory," Wikipedia, the free encyclopedia https://en.wikipedia.org/wiki/Chaos_theory

[2] D. Kuo, "Chaos and its computing paradigm," IEEE Potentials, April/May 2006, pp. 13-15.

[3] "Chaos computing," Wikipedia, the free encyclopedia

https://en.wikipedia.org/wiki/Chaos_computing 
[4] T. Munakata, S. Sinha, and W. L. Ditto, "Chaos computing: Implementation of fundamental logical gates by chaotic elements," IEEE Transactions on Circuits and Systems -I: Fundamental Theory and Applications, vol. 49, no. 11, November 2002, pp. 1629-1633.

[5] K. Murali, S. Sinha, and I. R. Mohamed, "Chaos computing: Experimental realization of NOR gate using a simple chaotic circuit." Physics Letters A, vol. 339, 2005, pp. 39-44.

[6] B. Kia, "Chaos computing: From theory to application," Doctoral Dissertation,

Arizona State University, December 2011.

[7] T.Munakata et al., "Chaos computing: A unified view," International Journal of Parallel, Emergent and Distributed Systems, vol. 25, no. 1, February 2010, pp. 3-16.

[8] B. Majumder et al., "Chaos computing for mitigating side channel attack," Proceedings on IEEE International Symposium on Hardware Oriented Security and Trust, April-May 2018, pp. 143-146.

[9] L. Kocarev, Z. Galias, and S. Lian (eds.), Intelligent Computing Based on Chaos. Berlin: Springer, 2009.

\section{ABOUT THE AUTHORS}

Matthew N.O. Sadiku is a professor at Prairie View A\&M University, Texas. He is the author of several books and papers. He is an IEEE fellow. His research interests include computational electromagnetics and computer networks.

Adebowale Shadare is a doctoral student at Prairie View A\&M University, Texas. He is the author of several papers.

Sarhan M. Musa is a professor in the Department of Engineering Technology at Prairie View A\&M University, Texas. He has been the director of Prairie View Networking Academy, Texas, since 2004. He is an LTD Spring and Boeing Welliver Fellow.



Figure 1 A typical circuit model for implementing NOR gate [5]. 